\title{
Design of interactive literacy activities for Deaf people on mobile devices
}

\author{
Paola L. Santollo-Vargas, Laura S. Gaytán-Lugo, Silvia B. Fajardo-Flores, \\ Pedro C. Santana-Mancilla
}

Published: 30 November 2021

\begin{abstract}
Reading and writing are daily communicative activities for our integration in society. For Deaf people, whose first language is sign language, these skills result complicated. In Mexico, there is a high percentage of Deaf people who have not acquired these skills, mainly due to the lack of trained personnel and educational guidance. Our proposal aims to design a mobile application with basic exercises to support the learning of reading and writing skills for the Deaf, using as a starting point their knowledge of the words in Mexican Sign Language (LSM, in Spanish). For the development of the proposal, we used the design thinking method, which includes the stages: empathize, define, devise, prototype. The low fidelity prototype was tested by an expert in LSM and teaching Spanish to deaf students. The results suggest that the activities are coherent and adequate for the Deaf user to appropriate the written words, and that the prototype has a clear flow.
\end{abstract}

\section{Keywords:}

Deaf; Accessibility; Interaction; Literacy; Reading and writing process.

\section{Introducción}

Las personas sordas cuya primera lengua es la lengua de señas generalmente tienen dificultades para adquirir habilidades de lectoescritura. Según el Laboratorio Nacional Diversidades [8], el $24 \%$ de las personas en México que presentan una discapacidad auditiva son analfabetas, de los cuales $55 \%$ cursan hasta el nivel básico, debido a que existe una limitación para encontrar maestros capacitados y escuelas acordes a sus necesidades de aprendizaje, así como orientación educativa oportuna. Aunado a esto, el español es para ellos una segunda lengua, muy diferente a la lengua de señas

Santollo-Vargas, Paola L., Gaytán-Lugo Laura S.

Facultad de Ingeniería Mecánica y Eléctrica

Universidad de Colima

Coquimatlán, México

psantollo@ucol.mx, laura@ucol.mx

Fajardo-Flores, Silvia B., Santana-Mancilla, Pedro C. Facultad de Telemática

Universidad de Colima

Colima, México

medusa@ucol.mx,psantana@ucol.mx
[3]. Por lo anterior, es importante ofrecer materiales educativos acordes a sus necesidades particulares.

Debido a sus posibilidades multimodales y a su actual pervasividad, las tecnologías representan una buena plataforma de apoyo al proceso enseñanza-aprendizaje a través de materiales educativos para este sector de la población. En este documento se presenta el diseño de ejercicios interactivos que sientan las bases para la adquisición de vocabulario escrito a usuarios sordos, tomando en cuenta sus características y los principios instruccionales adecuados a ellos. Los ejercicios están diseñados para una interfaz de dispositivo móvil, sin embargo, pueden ser implementados en otra plataforma, como Web o de escritorio.

\section{Enseñanza de la lectoescritura a personas sordas}

El atraso en la adquisición del lenguaje en personas sordas resulta en bajos niveles de alfabetización [7]. Los hijos sordos de padres sordos avanzan en la adquisición del lenguaje de manera similar a los niños oyentes de padres oyentes; son los niños sordos hijos de padres oyentes los que desarrollan el lenguaje más lentamente [6]. Son dichos niños los que generalmente experimentan dificultades de lectoescritura. La habilidad auditiva permite adquirir no solo sonidos, sino experiencias lingüísticas, conceptuales y sociales a las cuales las personas sordas no están expuestas, lo cual los aísla en muchos sentidos. Por otro lado, es común que los métodos de enseñanza utilizados con estudiantes sordos sean similares a los que se utilizan con oyentes, lo cual significa que están basados en competencias lingüísticas orales, de las cuales las personas sordas carecen [4].

Algunas de las dificultades comunes de la lectoescritura en los sordos son el reducido vocabulario y la falta de dominio de la morfosintaxis. El conocimiento de vocabulario es solo una de las condiciones necesarias para lograr la comprensión lectora; por otro lado, la cantidad de vocabulario adquirido por los niños sordos en edad escolar es inferior al de los niños oyentes [1], por lo cual es importante ofrecer herramientas de apoyo al aprendizaje de nuevas palabras escritas.

Para los estudiantes sordos, la enseñanza bilingüe proporciona un mejor acceso al lenguaje escrito, pues permite transferir habilidades lingüísticas. En una educación con enfoque bilingüe, se busca que los estudiantes sordos reflexionen a partir de su propia cultura para incorporar aspectos de la cultura oyente. Esto implica que la base para la construcción de su pensamiento sea la lengua de señas, mientras que el lenguaje escrito se utiliza como vehículo para 
entrar en contacto con la sociedad oyente [10]. Está comprobado que aquellos programas bilingües que además se respaldan en la lengua de señas obtienen mejores resultados que aquellos que solo usan el método puro [5].

Según Cruz Aldrete [3], un modelo educativo adecuado debe incluir tanto docentes sordos nativos para que instruyan que las señas aprendidas sean las correctas, como maestros oyentes que conozcan la lengua de señas con el fin de hacer del aula un espacio señante en donde pueden comunicar un mensaje de manera viso gestual. Con base en la educación bicultural-bilingüe, Torres López [10] propone utilizar el principio de comunicación total proximal para la enseñanza de lectoescritura a personas sordas, primeramente, presentando una imagen, seguida por el ideograma en lengua de señas, la dactilología, y finalmente la escritura.

Las tecnologías de información pueden ser utilizadas para apoyar el proceso de enseñanza-aprendizaje dentro de un modelo educativo bilingüe. A nivel Latinoamérica, existen algunas aplicaciones creadas con este fin: Sueñaletras, aplicación chilena desarrollada por el Centro de Desarrollo de Tecnologías de Inclusión (CEDETI), es un material de apoyo para la enseñanza de la lectoescritura en estudiantes sordos e hipoacúsicos, con la ayuda de videos usando la lengua de señas, actividades dactilológicas y la representación de lectura labial. El proyecto argentino DANE de tecnología para la inclusión desarrolló aplicaciones para niños con discapacidad, incluyendo algunas dirigidas a niños sordos con enfoque principalmente de comunicación con sus familiares oyentes, y aprendizaje de las vocales. DIELSEME es una aplicación interactiva basada en el Diccionario Español-Lengua de Señas Mexicana de la Secretaría de Educación Pública (SEP) de México, que incluye además de la definición, el video en lengua de señas mexicana (LSM) de las palabras. ALAS (Alfabetizar A Sordos) es una plataforma Web desarrollada por la Universidad Veracruzana, y tiene como propósito apoyar al proceso de lectoescritura del español.

Nuestra propuesta tiene por objetivo diseñar una aplicación móvil con ejercicios básicos de apoyo al aprendizaje de la lectoescritura para sordos, en un contexto bilingüe en el que la primera lengua es la LSM y la segunda es el español escrito.

\section{Desarrollo}

Para el desarrollo de la propuesta se utilizó el método design thinking [2], el cual es un proceso de diseño iterativo centrado en el usuario para resolver problemas de manera creativa, e incluye las etapas: empatizar, definir, idear, prototipar y probar. A continuación, se presenta el desarrollo del prototipo de aplicación considerando cada una de estas fases.

Empatizar. En esta etapa del proceso se busca conocer el tipo de usuario al cual está dirigida la aplicación a desarrollar, así como sus necesidades y su contexto. A fin de comprender el contexto y los principales problemas de lectoescritura de las personas sordas, lo ideal es tener un acercamiento directo que permita la observación de primera mano; sin embargo, existe la evidente limitación de las diferencias en comunicación sin la intervención de un intérprete de lengua de señas, sin embargo, existen alternativas. Como primer acercamiento, se realizó una entrevista no estructurada con la directora de la Asociación Manzanillense de Apoyo a Sordos I.A.P (AMAS), asociación civil fundada en 2006. Gracias a sus años de experiencia como fundadora y directora de AMAS, y además como madre de una persona sorda, la entrevistada nos dio información que nos permitió conocer el contexto, necesidades y situaciones que enfrentan los sordos en aspectos de comunicación, socialización y educativas. Entre los elementos relacionados a la lectoescritura, se encontró que las personas sordas tienen un vocabulario reducido, y que a pesar de que memorizan lenguaje escrito en forma de palabras, no necesariamente las entienden; es decir, es común que aprendan la palabra, pero no sepan cómo utilizarla.

También se obtuvieron algunos ejemplos de la manera en la que los sordos escriben:

- Mami donde tú

- Estoy en mi casa de Ana

- Mamá yo ir pasear

En el primer texto, la persona sorda pregunta a su mamá dónde está. En el segundo enunciado, se observa que, para referirse a la casa de Ana, dice " $m i$ casa", esto es porque Ana dice " $m i$ casa" al referirse a su casa. Mientras que en el tercer ejemplo es notable la disfunción sintáctica en la oración. Si bien los aspectos a considerar son diversos, uno de los que se puede atender de manera más inmediata es la adquisición de vocabulario.

Definir. Durante esta etapa se busca tomar en cuenta la información de contexto obtenida en la etapa anterior e identificar las áreas de oportunidad en las cuales se debe enfocar el proyecto para ofrecer una mejor solución a las necesidades del usuario.

Potter et al [9] explican que cuando se diseña para usuarios sordos se debe establecer el nivel en el que se encuentran y utilizar técnicas aptas para el mismo; en particular, mencionan que debe utilizarse un lenguaje que les sea familiar para retener su atención. De acuerdo con la literatura presentada, se decidió utilizar un enfoque bilingüe tomando como punto de partida la LSM para introducir las palabras nuevas.

Nuestra propuesta está dirigida a personas sordas con conocimiento básico de lengua de señas mexicana (LSM), ya que se utilizará como base para el aprendizaje de la palabra escrita. Debido a que las personas sordas a quienes va dirigida esta aplicación están aprendiendo el español escrito, es importante reducir al máximo la cantidad de texto que se presente, además de utilizar imágenes de apoyo para complementar el texto.

Como meta instruccional se definió el aprendizaje de la escritura de los días de la semana en español. Los objetivos instruccionales son: (1) Identificar la morfología de la palabra escrita de los días de la semana; y (2) Crear una vinculación entre la señal de la palabra ya conocida con la palabra en español escrito, involucrando además la creación de un vínculo entre las letras que conforman la palabra escrita con la seña de cada una. Esta primera meta instruccional es la base para el diseño de los ejercicios, los cuales, una vez evaluados y mejorados podrán utilizarse para el aprendizaje de la lectoescritura de otras palabras.

Idear. Propuesta de diseño de la solución. Se partió de la representación de los días de la semana en LSM que ya es familiar al usuario(a), así como su representación dactilológica, como base para el aprendizaje de la palabra escrita. Las actividades propuestas se presentan en secuencia, aumentando de dificultad en cada actividad:

- Demostración: Actividad introductoria que consiste en la presentación de los videos en LSM de cada día, y la palabra escrita correspondiente (Figura 2a).

- Lectura e identificación: Consiste en un ejercicio en el cual el usuario(a) observa el video en LSM de un día de la semana, y debe seleccionar la palabra a la cual corresponde (Figura 2b). 
- Demostración dactilológica: Se muestra cada letra de la palabra, así como la seña correspondiente (Figura 2c).

- Completar la palabra: Se presenta la palabra de los días de la semana escrita parcialmente, a partir de la cual el usuario(a) deberá completar las letras faltantes. Inicialmente se le indicará la seña correspondiente a la letra, y conforme las palabras se vayan presentando la dificultad aumentará, es decir, el número de letras faltantes aumentará (Figura 3a,b,c,d).

- Escribir la palabra sin soporte: Finalmente, se busca que el usuario(a) escriba por sí solo(a) la palabra completa a partir de la seña de la palabra (Figura 3e).

La idea del diseño conceptual se trabajó primeramente a nivel de sketch, incluyendo el comportamiento propuesto para cada actividad (Figura 1). Las instrucciones se dan utilizando la menor cantidad de palabras posibles; en nuestro caso un verbo que representa la acción a realizar, acompañado de una imagen representativa de la acción. Por ejemplo, la indicación "Observa" acompañada por la imagen de un ojo. La retroalimentación a cada ejercicio se da utilizando la palabra escrita "Correcto" o "Incorrecto", acompañada por una estrella y una equis respectivamente.

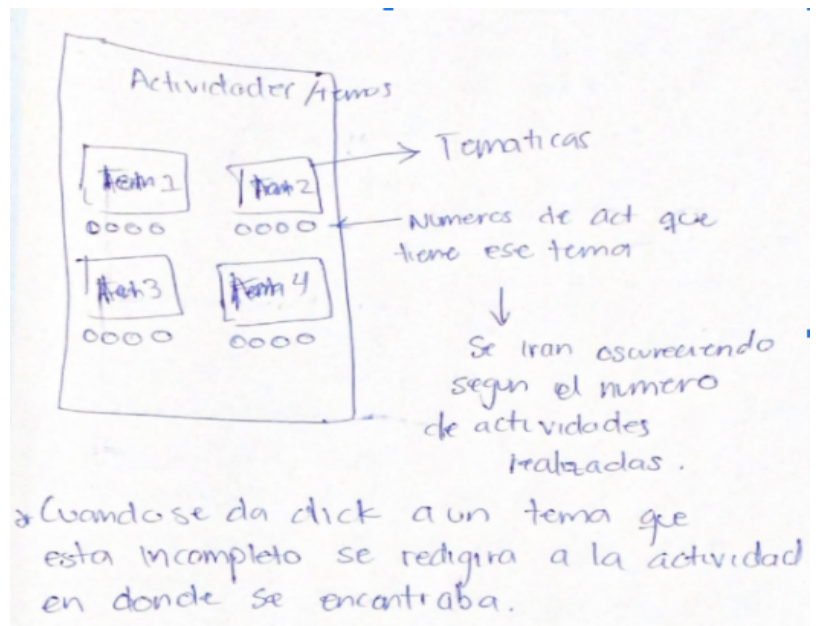

Figura 1. Primer sketch del diseño conceptual (Elaboración propia).

Prototipar. Con base en las ideas de diseño obtenidas en la etapa anterior se crea el prototipo de baja fidelidad, es decir, un prototipo no funcional el cual puede ser probado con algún método de inspección o bien con usuarios, a fin de obtener retroalimentación que nos permita mejorar el diseño en la siguiente iteración. El prototipo de baja fidelidad se realizó en la herramienta Figma. Como parte de los recursos multimedia se incluyen videos de cada día de la semana en Lengua de Señas Mexicana (LSM), para lo cual se utilizaron los videos del proyecto wikisigns.org, así como imágenes con la dactilología de la palabra. A continuación, se muestran algunas interfaces del prototipo de baja fidelidad:

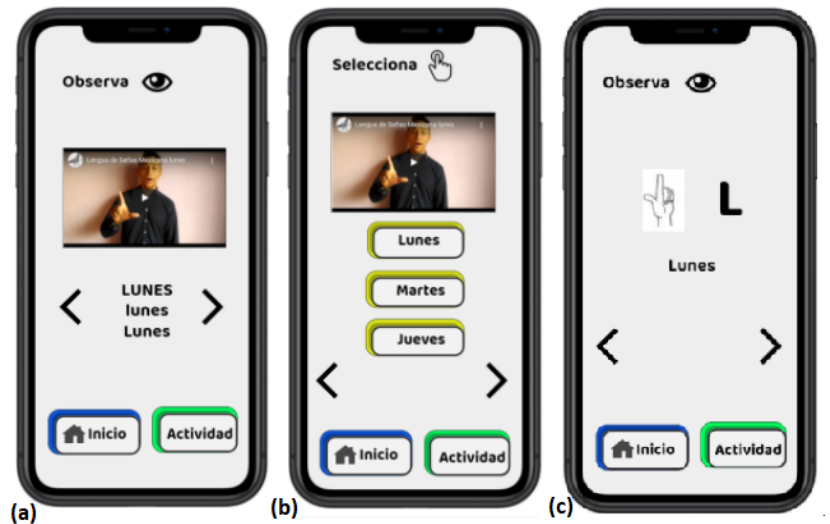

Figura 2. (a) Actividad introductoria de demostración, (b) Actividad de lectura e identificación, (c) Demostración dactilológica (Elaboración propia).

Probar. En esta primera iteración, el prototipo de baja fidelidad fue evaluado por una experta en LSM y enseñanza de español escrito a estudiantes sordos, quien actualmente trabaja en un Centro de Atención Múltiple (CAM) del estado de Colima, y cuenta con más de 25 años de experiencia en la enseñanza de español a personas sordas. El propósito de esta evaluación fue inspeccionar qué tan apropiado es el diseño de las actividades de lectoescritura con respecto a:

1) La manera en que se presentan los días de la semana.

2) La manera de dar las instrucciones.

3) La secuencia de las actividades.

4) La conveniencia de las actividades.

5) La manera de dar retroalimentación a las respuestas.

Se diseñó un cuestionario con cinco preguntas abiertas, correspondientes a cada uno de los aspectos a inspeccionar. La inspección se hizo de manera virtual y asíncrona. Se hizo llegar a la experta el wireflow del prototipo a fin de que observara la secuencia de las diferentes actividades; asimismo se le pidió que contestara por escrito el cuestionario.

Como resultados de esta primera inspección, se obtuvo lo siguiente:

1) La experta comentó que la presentación le parece adecuada; al respecto sugirió que sea una persona sorda quien realice la seña.

2) Respecto a la manera de dar instrucciones, la experta mencionó que le parece interesante la secuencia que se utiliza para introducir la seña y la grafía; sin embargo, es necesario revisar la posición de las manos, puesto que, en el ejemplo del día lunes, la mano se encuentra al revés, por lo que es necesario estar seguros de que sean correctas.

3) El diseño de esta actividad le parece adecuado, ya que le permite al sordo ver primeramente la seña que ya conoce, para después asociarla con la grafía.

4) Las actividades le resultan coherentes y le parecen propias para aumentar gradualmente la complejidad. Considera que las actividades permitirán al alumno apropiarse de la palabra escrita y, a su vez, el deletreo en su lengua.

5) Considera que el flujo de las acciones es claro, así como la forma en la que se brinda retroalimentación al usuario. 


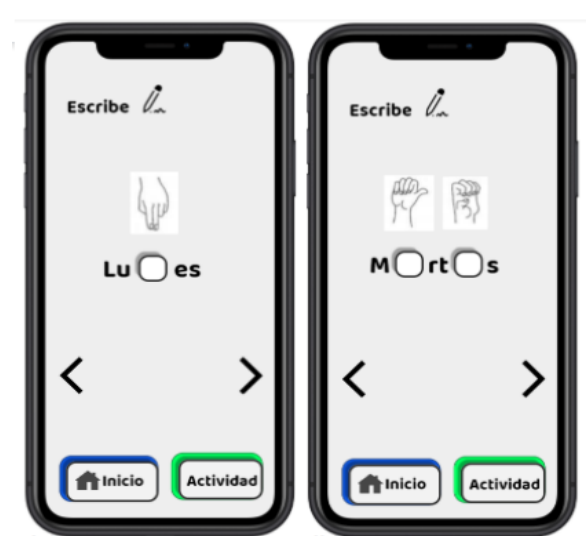

(a) (b)

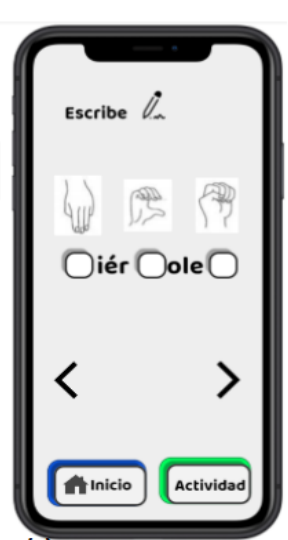

(c)

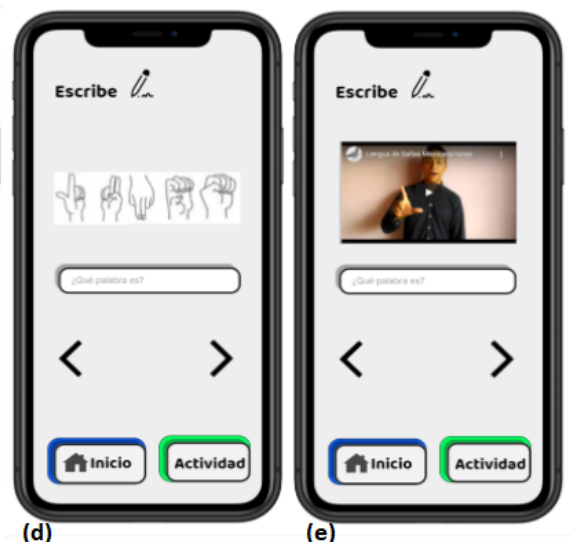

(e)

Figura 3. (a,b,c,d) Actividades de completar la palabra, (e) Actividad de escritura sin soporte (Elaboración propia).

Si bien la retroalimentación de la experta es útil como parte de la inspección de un primer prototipo no funcional, es necesario realizar una evaluación con usuarios sordos en una segunda iteración, para obtener retroalimentación de primera mano sobre las características incluidas en el diseño.

\section{Conclusiones}

En este documento se presenta una propuesta de actividades interactivas de lectoescritura dirigidas a personas sordas que conocen la LSM, en un prototipo de baja fidelidad para dispositivos móviles. La relevancia de esta propuesta no consiste únicamente en ser una aplicación dirigida a usuarios sordos, sino que está basada en un diseño instruccional de acuerdo a los objetivos de aprendizaje planteados, está alineado con el aprendizaje bajo el modelo bilingüe bicultural, el cual toma como base la lengua de señas para adquirir el vocabulario escrito, y finalmente, fue desarrollado siguiendo un proceso centrado en el usuario. Actualmente hemos finalizado la primera iteración del diseño siguiendo un proceso centrado en el usuario, y contamos con los resultados de una primera evaluación por una experta en LSM y enseñanza de español escrito a personas sordas. A partir de los resultados de la inspección por la experta se realizarán las mejoras a fin de construir un prototipo funcional que será evaluado por usuarios en la segunda iteración. Una vez mejorado el diseño de la interfaz y verificado el alcance de los objetivos instruccionales, las actividades pueden utilizarse para el aprendizaje de nuevas palabras a nivel introductorio, enriqueciendo así los contenidos de la aplicación. Es importante mencionar que además del aprendizaje de las palabras escritas, son necesarios ejercicios adicionales que permitan a los usuarios sordos observar y practicar el uso de las palabras aprendidas en diferentes contextos, para reducir su déficit experiencial.

\section{Referencias}

[1] José María Augusto, José Antonio Adrián, Jesús Alegría, and Rosa Martínez. 2002. Dificultades lectoras en niños con sordera. Psicothema 14, 4: 746-753. Recuperado desde https://www.redalyc.org/articulo.oa?id=72714409.
[2] Tim Brown. 2008. Design thinking. Harvard business review, 86,6 .

[3] Miroslava Cruz-Aldrete. 2017. Reflexiones sobre la Educación Bilingüe Intercultural para el sordo en México. Recuperado http://repositoriocdpd.net:8080/handle/123456789/1802

[4] Rafaela Gutiérrez Cáceres. 2004. Cómo escriben los alumnos sordos. Ediciones Aljibe. Málaga.

[5] Valeria Herrera, Jesús M. Alvarado, Anibal Puente, Alfredo Ardilla. 2007. Códigos de lectura en sordos: La dactilología y otras estrategias visuales y kinestésicas. Revista latinoamericana de psicología, 39, 2, 269-286. Recuperado desde

http://publicaciones.konradlorenz.edu.co/index.php/rlpsi/artic le/view/320

[6] Nobuo Masataka. 2000. The role of modality and input in the earliest stage of language acquisition: Studies of Japanese Sign Language. Language acquisition by eye: 3-24.

[7] Connie Mayer. 2007. What Really Matters in the Early Literacy Development of Deaf Children. Journal of Deaf Studies and Deaf Education 12, 4: 411-431. https://doi.org/10.1093/deafed/enm020

[8] Laboratorio Nacional Diversidades (2018). La desigualdad en personas con discapacidad auditiva. Recuperado desde https://www.animalpolitico.com/diversidades-fluidas/ladesigualdad-en-las-personas-con-discapacidad-auditiva/

[9] Leigh Ellen Potter, Jessica Korte, and Sue Nielsen. 2014. Design with the deaf. In Proceedings of the 2014 conference on Interaction design and children, 249-252. https://doi.org/10.1145/2593968.2610464

[10] David Torres López. 2011. Estrategias y recursos para el desarrollo de competencias en el niño sordo, Modelo de educación intercultural bilingüe. Kofo ediciones.

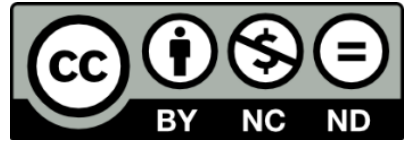

(C) 2021 by the authors. This work is licensed under the Creative Commons AttributionNonCommercial-NoDerivatives 4.0 International License. To view a copy of this license, visit http://creativecommons.org/licenses/by-nc-nd/4.0/ or send a letter to Creative Commons, PO Box 1866, Mountain View, CA 94042, USA. 